\title{
Easy-to-learn cardiopulmonary resuscitation training programme: a randomised controlled trial on laypeople's resuscitation performance
}

\author{
Rachel Jia Min $\underline{K o}^{1}, \mathrm{RN}, \mathrm{BSN}(\mathrm{Hons})$, Swee Han $\underline{\mathrm{Lim}}^{2}$, FRCSE, FRCPE, Vivien Xi $\underline{W u^{3}}$, RN, PhD, Tak Yam Leong${ }^{4}$, BEng,
} Sok Ying Liaw $^{3}$, RN, PhD

INTRODUCTION Simplifying the learning of cardiopulmonary resuscitation (CPR) is advocated to improve skill acquisition and retention. A simplified CPR training programme focusing on continuous chest compression, with a simple landmark tracing technique, was introduced to laypeople. The study aimed to examine the effectiveness of the simplified CPR training in improving lay rescuers' CPR performance as compared to standard CPR.

METHODS A total of 85 laypeople (aged 21-60 years) were recruited and randomly assigned to undertake either a twohour simplified or standard CPR training session. They were tested two months after the training on a simulated cardiac arrest scenario. Participants' performance on the sequence of CPR steps was observed and evaluated using a validated CPR algorithm checklist. The quality of chest compression and ventilation was assessed from the recording manikins. RESULTS The simplified CPR group performed significantly better on the CPR algorithm when compared to the standard CPR group $(p<0.01)$. No significant difference was found between the groups in time taken to initiate CPR. However, a significantly higher number of compressions and proportion of adequate compressions was demonstrated by the simplified group than the standard group $(p<0.01)$. Hands-off time was significantly shorter in the simplified CPR group than in the standard CPR group $(p<0.001)$.

CONCLUSION Simplifying the learning of CPR by focusing on continuous chest compressions, with simple hand placement for chest compression, could lead to better acquisition and retention of CPR algorithms, and better quality of chest compressions than standard CPR.

Keywords: bystander CPR, cardiac arrest, cardiopulmonary resuscitation, chest compression

\section{INTRODUCTION}

Bystander cardiopulmonary resuscitation (CPR) is crucial in the 'chain of survival' in order to improve the survival outcome of outof-hospital cardiac arrest (OHCA). ${ }^{(1)}$ The prevalence of bystander $\mathrm{CPR}$ remains relatively low worldwide, with a reported range of $1 \%-44 \%{ }^{(2,3)}$ Barriers faced by bystanders to performing CPR were identified in previous studies, including concerns regarding possible disease transmission during mouth-to-mouth (MTM) ventilation, poor skills retention, a lack of confidence and a fear of legal implication. ${ }^{(4,5)}$

In an effort to increase bystander CPR rates, the requirement of MTM ventilation was reviewed in CPR guidelines. ${ }^{(6,7)}$ Given the supporting evidence that the application of continuous chest compression CPR was at least as good as standard CPR, ${ }^{(8)}$ continuous chest compression CPR was recommended as an alternative method for those who are unable or unwilling to perform MTM. ${ }^{(9,10)}$ However, given that this is only an alternative method for CPR, the learning of standard CPR that includes MTM ventilation has remained unchanged. Another aspect of psychomotor learning in bystander CPR that remains unchanged despite existing evidence is the method of locating the landmark for chest compression. ${ }^{(11)}$ The conventional method of locating the landmark with one fingerbreadth above the xiphoid process, by tracing the lower margin of the victim's rib cage, was found to prolong interruptions of chest compressions compared to the placement of the heel of the hand over the centre of the chest. ${ }^{(12)}$

A major obstacle to achieving public proficiency in CPR is poor acquisition and retention of the necessary knowledge and skills. ${ }^{(13-15)}$ The complex psychomotor tasks involved in the learning of bystander CPR have prompted the need to simplify CPR training procedures. Applying the cognitive load theory, simplifying the steps to be learned could help to reduce the cognitive load imposed on the learner's working memory, and thereby aid learning and memory. ${ }^{(16)}$ Considering the importance of delivering high-quality chest compressions, it would be reasonable to simplify CPR training by focusing lay rescuers' learning on continuous chest compressions, with a simple landmark tracing technique for chest compression. In this study, we aimed to determine whether the simplification of psychomotor skills involved in CPR would lead to better acquisition of the CPR algorithm and sustain the quality of CPR performance in the long term.

${ }^{1}$ National Heart Centre Singapore, ${ }^{2}$ Department of Emergency Medicine, Singapore General Hospital, ${ }^{3}$ Alice Lee Centre for Nursing Studies, NUS Yong Loo Lin School of Medicine, National University of Singapore, ${ }^{4}$ SingHealth Duke-NUS Breast Centre, Singapore

Correspondence: Dr Sok Ying Liaw, Associate Professor, Alice Lee Centre for Nursing Studies, NUS Yong Loo Lin School of Medicine, National University of Singapore, Level 2 , Clinical Research Centre, Block MD11, 10 Medical Drive, Singapore 117597.nurliaw@nus.edu.sg 


\section{METHODS}

A prospective randomised controlled trial with a post-test design was conducted at the Centre for Healthcare Simulation, National University of Singapore (NUS) Yong Loo Lin School of Medicine, Singapore, from November 2013 to January 2014. Ethical approval was obtained from the university's institutional review board. Participants were recruited via convenience sampling using recruitment posters, which were put up in public accessible areas. The target population was laypeople who were aged 21-60 years and possessed a minimum educational qualification of General Certificate of Education (GCE) Ordinary Level (O-level). A wide age range was chosen so that it lay within the age range of a typical bystander in the community. Only participants with a minimum of GCE O-level educational qualification were eligible, as the teaching contents used in the study required a minimum of elementary level comprehension. Participants were excluded if they had been diagnosed with any medical condition or were pregnant. The findings of a previous study were used to estimate the required sample size. ${ }^{(17)}$ Using an effect size of 0.63 , power analysis suggested that at least 35 participants in each group were required to achieve $80 \%$ power at $5 \%$ level of significance. Taking into consideration possible attrition, 90 participants were recruited, with 45 in each group.

Out of a total of 90 interested participants, 85 participants met the eligibility criteria and gave their consent. The participants were randomly assigned to either the simplified or standard CPR group using computer-aided randomisation stratified by gender and age (under or over 40 years). Gender and age have been shown to be factors affecting the quality of CPR. ${ }^{(18,19)}$ The use of stratified randomisation ensured that the groups were comparable with regard to their specific demographic characteristics, thereby reducing known variability. ${ }^{(20)}$ The grouping allocation was concealed from the participants.

Participants in the simplified and standard CPR groups underwent a two-hour CPR training session. Both CPR training programmes were taught by Basic Cardiac Life Support (BCLS)certified instructors using the Resusci Anne ${ }^{\circledR}$ SkillReporter ${ }^{\mathrm{TM}}$ manikins (Laerdal Medical AS, USA). Prior to the training, a briefing session was conducted for the BCLS instructors on the two types of CPR training. A teaching guide containing the CPR techniques (Table I) was prepared for the instructors. The modes of delivery for both groups were based on the

Table I. Teaching guide for standard and simplified CPR.

\begin{tabular}{|c|c|}
\hline Standard CPR & Simplified CPR \\
\hline \multicolumn{2}{|l|}{ Check for danger } \\
\hline $\begin{array}{l}\text { Assess responsiveness } \\
\text { - Tap or gently shake the victim } \\
\text { - Call loudly "Hello! Hello! Are you OK?" }\end{array}$ & \\
\hline \multicolumn{2}{|c|}{$\begin{array}{l}\text { Shout for help if the victim is unresponsive. If no response, activate EMS by dialling ambulance number ' } 995 \text { ', get AED if there is one } \\
\text { nearby and return to the victim }\end{array}$} \\
\hline \multicolumn{2}{|l|}{ Position the victim } \\
\hline \multicolumn{2}{|l|}{ Open the airway: perform head tilt-chin lift manoeuvre } \\
\hline \multicolumn{2}{|c|}{$\begin{array}{l}\text { Assess breathing } \\
\text { - Check for normal breathing: look, listen and feel }(10 \mathrm{sec}) \\
\text { - Place ear and cheek over the victim's mouth and nose, and look for chest rise and fall } \\
\text { - Listen for air escaping during exhalation and feel for the flow of air }\end{array}$} \\
\hline \multicolumn{2}{|c|}{ Locate the landmark for chest compression } \\
\hline \multicolumn{2}{|c|}{ Proper compression technique } \\
\hline $\begin{array}{l}\text { - Depress the sternum (at least } 50 \mathrm{~mm} \text { ) } \\
\text { - Say mnemonic [ } 1 \text { and } 2 \text { and } 3 \text { and } 4 \text { and } 5 \text { and } 1 \text { and } 2 \text { and } 3 \\
\text { and } 4 \text { and } 10 \text { and... 30] } \\
\text { - Chest compression rate is at least } 100 / \mathrm{min}\end{array}$ & $\begin{array}{l}\text { - Depress the sternum (at least } 50 \mathrm{~mm} \text { ) } \\
\text { - Say mnemonic [ } 1 \text { and } 2 \text { and } 3 \text { and } 4 \text { and } 5 \text { and } 1 \text { and } 2 \text { and } 3 \text { and } 4 \\
\text { and } 10 \text { and... 100] } \\
\text { - Chest compression rate is at least } 100 / \mathrm{min}\end{array}$ \\
\hline $\begin{array}{l}\text { Perform two mouth-to-mouth breaths after every } 30 \text { chest } \\
\text { compressions }\end{array}$ & Rest $10 \mathrm{sec}$ after every 100 chest compressions \\
\hline \multicolumn{2}{|l|}{ - Mouth-to-mouth breathing } \\
\hline \multicolumn{2}{|l|}{ - Give two breaths (1 sec/breath; $400-600 \mathrm{~mL}$ of air per breath) } \\
\hline \multicolumn{2}{|l|}{ - Allow lung deflation between each breath } \\
\hline $\begin{array}{l}\text { Perform five cycles of } 30 \text { chest compressions and two } \\
\text { ventilations }\end{array}$ & Perform two cycles of 100 chest compressions \\
\hline
\end{tabular}

AED: automated external defibrillator; CPR: cardiopulmonary resuscitation; EMS: emergency medical services 


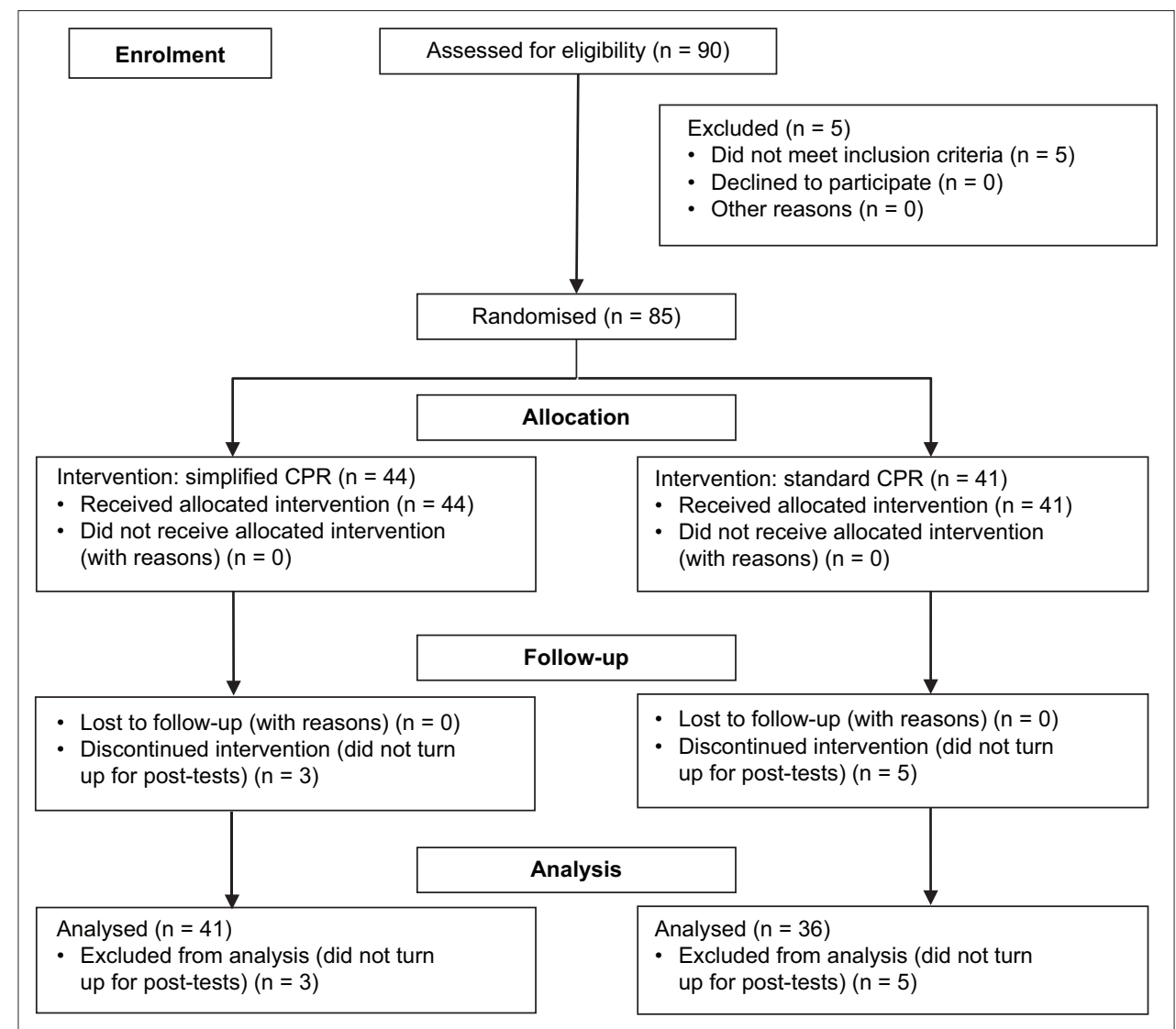

Fig. 1 Flowchart shows the process of data collection. CPR: cardiopulmonary resuscitation

existing citizen CPR programme, which involved approximately 90 minutes of demonstration and practice, and 30 minutes of return demonstration and feedback. ${ }^{(21)}$ The instructor-toparticipant ratio was 1:6, which was based on the American Heart Association recommendation. While the simplified CPR group underwent the simplified CPR training, the standard CPR group received standard CPR training. Using the existing citizen CPR programme guidelines, ${ }^{(21)}$ participants in the standard CPR group were taught compressions and MTM ventilation CPR. The participants in the simplified CPR group were taught continuous chest compressions (with no MTM ventilations) and a simple landmark tracing technique for locating chest compression. Table I shows the descriptions of the CPR techniques taught to the simplified and standard CPR groups.

About two months following the training, participants from both groups undertook post-tests in a simulated scenario at a university simulation laboratory. A total of eight participants three from the simplified CPR group and five from the standard CPR group - did not turn up for the post-tests (Fig. 1). In the posttests, a scenario of a woman who suddenly collapsed on the floor in a shopping mall was presented to the individual participants. The recording manikin used was dressed in a wig and made to look like a woman in a long dress, with glycerine smeared around the mouth to simulate saliva secretions. Before the simulation post-test, each participant was given a brief synopsis of the scenario and instructed to perform the tasks that they would do in real life. During the simulation tests, the assessors acted as passers-by who observed and rated the participant's performance using the CPR algorithm checklist. A research assistant recorded the time taken by the participants to initiate CPR, starting from the beginning of the scenario until the time the first compression was initiated. Each test lasted eight minutes before the participants were informed that help had arrived. The participant's CPR performance was recorded by the Resusci Anne ${ }^{\circledR}$ SkillReporter ${ }^{\mathrm{TM}}$ manikins. The entire simulation test was recorded on video.

A CPR algorithm, which is a seven-item checklist (Table II), was adapted and modified from the Cardiff test (V3.1) to evaluate the participant's performance when recognising a cardiac arrest event and carrying out actions in a correct sequence. ${ }^{(22)}$ The Cardiff test (V3.1) was developed to assess CPR and automated external defibrillation performance from analyses of video recordings and data drawn from a recording strip attached to a manikin. ${ }^{(22)}$ The total score for the CPR algorithm checklist was calculated by adding the scores for the seven items, with a maximum possible score of 25. Content validity was established by a panel of experts, including a member of National Resuscitation Council, Singapore, and three experienced BCLS instructors. An intra-class correlation coefficient, across two raters, of 0.93 (95\% confidence interval 0.80-0.98) was obtained in our study, which indicated the excellent inter-rater reliability of the CPR algorithm checklist.

Variables measured by the Resusci Anne ${ }^{\circledR}$ SkillReporter ${ }^{\mathrm{TM}}$ manikins included average compression depth, number of compressions per minute, total number of compressions, total number of adequate compressions ( $\geq 50 \mathrm{~mm}$ ), hands-off time, 
Table II. Cardiopulmonary resuscitation algorithm checklist.

\begin{tabular}{|c|c|c|}
\hline Step & Status of action & \\
\hline 1. Assess for danger & $\square$ Performed & $\square$ Not performed \\
\hline \multicolumn{3}{|l|}{ 2. Check responsiveness } \\
\hline $\begin{array}{l}\text { (i) Tap or shake the victim's shoulders } \\
\text { gently }\end{array}$ & $\begin{array}{l}\square \text { Performed adequately } \\
\square \text { Potentially dangerous }\end{array}$ & $\square$ Not performed \\
\hline (ii) Shout “Hello! Hello! Are you OK?" & $\square$ Performed & $\square$ Not performed \\
\hline 3. Shout "Call ambulance, 995" & $\begin{array}{l}\square \text { Call for ambulance - } 995 \\
\square \text { Get help - unspecified }\end{array}$ & $\begin{array}{l}\square \text { Call for ambulance - other number } \\
\text { Specify number: } \\
\square \text { Not performed }\end{array}$ \\
\hline 4. Open airway, perform head tilt-chin lift & $\begin{array}{l}\square \text { Performed } \\
\square \text { Visibly attempted }\end{array}$ & $\begin{array}{l}\square \text { Performed - other or maintained } \\
\square \text { Not performed }\end{array}$ \\
\hline \multicolumn{3}{|l|}{ 5. Assess breathing } \\
\hline Look & $\begin{array}{l}\square \text { Correct } \\
\square \text { Ineffective }\end{array}$ & $\square$ Not attempted \\
\hline Listen and feel & $\begin{array}{l}\square \text { Correct } \\
\square \text { Ineffective }\end{array}$ & $\square$ Not attempted \\
\hline 6. Perform chest compression & $\square$ Performed at least one compression & $\square$ Did not perform compression at all \\
\hline 7. Sequence performed in correct order & $\square$ Performed in correct order & $\square$ Performed in incorrect order. Please specify \\
\hline
\end{tabular}

proportion of adequate compressions, number of total ventilations and average inflation volume. These variables were measured by the assessors from the recording strips. The proportion of adequate compressions was calculated as the actual number of adequate compressions over the total number of compressions performed. Adequate chest compression was defined as compressions with a depth $\geq 50 \mathrm{~mm}$ in accordance with international guidelines. ${ }^{(21}$

Descriptive statistics were used to determine any differences in demographic characteristics between groups. Chi-square test or Fisher's exact test was computed for categorical variables (e.g. gender and ethnicity), while independent $t$-test was used for continuous variables (e.g. age). Analysis of covariance was conducted to compare the post-test scores between the two CPR groups, with adjustments for age and gender.

\section{RESULTS}

Out of the 85 participants recruited, 44 were allocated to the simplified CPR group and 41 to the standard CPR group. After excluding those who did not attend the post-tests, $41(93.2 \%)$ participants from the simplified CPR group and 36 (87.8\%) participants from the standard CPR group completed the study. There were no significant differences in demographic characteristics between the two groups (Table III), indicating homogeneity among the participants.

As a result of technical faults, chest compressions performed by four participants (simplified CPR, $\mathrm{n}=1$; standard CPR, $\mathrm{n}=$ 3 ) were not captured by the recording manikins. Hence, the chest compression performance of 40 and 33 participants in the simplified and standard CPR groups, respectively, were evaluated. The participants in the simplified CPR group had a significantly higher CPR algorithm score than those in the standard CPR group $(21.68 \pm 2.78$ vs. $19.22 \pm 3.67 ; p<0.01)$ (Table IV). No significant difference in CPR algorithm score was found between participants with tertiary and non-tertiary education within each group.

There was no significant difference in the time taken to initiate CPR between the simplified CPR and standard CPR groups (48.56 \pm 21.76 seconds vs. $57.53 \pm 38.48$ seconds; $p=0.34$ ). The simplified CPR group demonstrated a significantly higher number of compressions per minute than the standard CPR group (76.45 \pm 26.28 vs. $53.55 \pm 16.91 ; \mathrm{p}<0.001)$. A significantly higher total number of compressions was also demonstrated by the simplified CPR group when compared to the standard CPR group (443.28 \pm 17.76 vs. $353.09 \pm 124.07 ; p<0.01$ ) during the eight-minute test duration. The number of compressions with adequate depth was significantly higher in the simplified CPR group than the standard CPR group (130.53 \pm 174.23 vs. $33.79 \pm 64.16 ; p<0.01)$. There was no significant difference in depth of compressions between the simplified and standard CPR groups $(36.78 \pm 15.17 \mathrm{~mm}$ vs. $33.24 \pm 11.31 \mathrm{~mm} ; \mathrm{p}=0.10$ ). When comparing the proportion of adequate compressions, the simplified CPR group delivered a significantly higher proportion of adequate compressions than the standard CPR group $(27.74 \% \pm 34.90 \%$ vs. $9.41 \% \pm 18.76 \%$; $\mathrm{p}<0.01$ ).

Hands-off time was significantly shorter in the simplified CPR group than the standard CPR group $(88.35 \pm 52.84$ seconds vs. $198.52 \pm 60.00$ seconds; $p<0.001)$. This implied that in an eight-minute time period, a participant performing standard CPR paused for an average of three minutes to perform ventilation, whereas a participant performing simplified CPR would only pause to rest for one-third of that time.

13 out of 33 participants (39.39\%) from the standard CPR group did not attempt to perform any MTM ventilation. Among those who performed ventilation, the average inflation volume achieved by more than half of the participants (57.14\%) was either too little $(<400 \mathrm{~mL})$ or too much $(>600 \mathrm{~mL})$.

\section{DISCUSSION}

This study provided evidence that supports the use of a simplified CPR training programme for improved CPR algorithm learning and performance. A lack of realism in the test scenario was acknowledged by previous studies. ${ }^{17,23,24)}$ In our study, much effort was directed at enhancing the realism of the simulated OHCA to 
Table III. Demographic characteristics of participants.

\begin{tabular}{|c|c|c|c|c|}
\hline \multirow[t]{2}{*}{ Variable } & \multicolumn{3}{|c|}{ No. (\%) } & \multirow[t]{2}{*}{ p-value } \\
\hline & Total $(n=85)$ & Simplified CPR $(n=44)$ & Standard CPR $(n=41)$ & \\
\hline Age (yr)* & $30.78 \pm 12.05$ & $30.00 \pm 11.55$ & $31.61 \pm 12.65$ & 0.54 \\
\hline Gender & & & & 0.90 \\
\hline Men & $40(47.1)$ & $21(47.7)$ & $19(46.3)$ & \\
\hline Women & $45(52.9)$ & $23(52.3)$ & $22(53.7)$ & \\
\hline Chinese & $82(96.5)$ & $43(97.7)$ & $39(95.1)$ & \\
\hline Indian & $1(1.2)$ & $0(0)$ & $1(2.4)$ & \\
\hline Malay & $2(2.4)$ & $1(2.3)$ & $1(2.4)$ & \\
\hline Education level & & & & 0.74 \\
\hline GCE O-level & $12(14.1)$ & $5(11.4)$ & $7(17.1)$ & \\
\hline GCE A-level & $36(42.4)$ & $21(47.7)$ & $15(36.6)$ & \\
\hline Diploma & $2(2.4)$ & $1(2.3)$ & $1(2.4)$ & \\
\hline Degree & $35(41.2)$ & $17(38.6)$ & $18(43.9)$ & \\
\hline Prior training & & & & 0.76 \\
\hline Yes & $12(14.1)$ & $7(15.9)$ & $5(12.2)$ & \\
\hline No & $73(85.9)$ & $37(84.1)$ & $36(87.8)$ & \\
\hline Prior encounter & & & & 1.00 \\
\hline Yes & $1(1.2)$ & $1(2.3)$ & $0(0)$ & \\
\hline No & $84(98.8)$ & $43(97.7)$ & $41(100)$ & \\
\hline
\end{tabular}

* Data presented as mean \pm standard deviation. CPR: cardiopulmonary resuscitation; GCE: General Certificate of Education

Table IV. Cardiopulmonary resuscitation (CPR) performance of participants.

\begin{tabular}{|c|c|c|c|c|}
\hline \multirow[t]{2}{*}{ Variable } & \multicolumn{2}{|c|}{ Mean \pm standard deviation } & \multirow[t]{2}{*}{ F value } & \multirow[t]{2}{*}{ p-value } \\
\hline & Simplified CPR $(n=40)$ & Standard CPR $(n=33)$ & & \\
\hline CPR algorithm score & $21.68 \pm 2.78$ & $19.22 \pm 3.67$ & 8.7 & $<0.01$ \\
\hline Time to first compression (s) & $48.56 \pm 21.76$ & $57.53 \pm 38.48$ & 0.9 & 0.34 \\
\hline Compression per min & $76.45 \pm 26.28$ & $53.55 \pm 16.91$ & 23.9 & $<0.001$ \\
\hline Hands-off time (s) & $88.35 \pm 52.84$ & $198.52 \pm 60.00$ & 63.8 & $<0.001$ \\
\hline Total no. of adequate compressions & $130.53 \pm 174.23$ & $33.79 \pm 64.16$ & 11.8 & $<0.01$ \\
\hline Proportion of adequate compressions (\%) & $27.74 \pm 34.90$ & $9.41 \pm 18.76$ & 9.7 & $<0.01$ \\
\hline Average depth (mm) & $36.78 \pm 15.17$ & $33.24 \pm 11.31$ & 2.9 & 0.10 \\
\hline
\end{tabular}

assess participant performance under more realistic conditions. To mimic a realistic OHCA event, the recording manikin in our study was dressed as a woman in a wig and long dress, with glycerine smeared around her mouth to simulate saliva secretions. The test duration was set to be eight minutes, so as to better mimic the local ambulance response time.

Our study showed that participants in the simplified CPR group performed significantly better on the CPR algorithm than those in the standard CPR group two months after training. Although CPR learning retention was not evaluated in our study, previous studies have reported consistent findings on the deterioration of CPR learning over time. ${ }^{(17,25)}$ Despite the potential of learning deterioration in both forms of $\mathrm{CPR}$, our study supports the long-term effectiveness of simplified CPR training compared to standard CPR for facilitating the learning and retention of CPR steps. In simplified CPR, the learning of continuous chest compressions without MTM ventilations and a one-step landmark tracing technique reduced the required number of psychomotor steps. This would help to promote better acquisition and retention of learnt skills. Conversely, the considerable number of psychomotor skills to be learnt and performed in the standard CPR algorithm placed unrealistic demands on the learning curve of bystanders. ${ }^{(26)}$ According to Riegel et al, simplifying the current standard CPR may allow laypeople to learn and remember the vital steps and actions that could help save a life in the event of a real emergency. ${ }^{(27)}$

For this study, we postulated that being able to recall the simplified CPR algorithm could have translated into a reduction in the time taken to initiate compression. Although the simplified CPR group took an average of about nine seconds less to initiate compression, the difference was not significant when compared to the standard CPR group. Nevertheless, given that every one-minute delay can reduce a victim's survival rate by $7 \%-10 \%$, the longer time taken by participants performing standard CPR to initiate compression could be clinically meaningful. Our finding is inconsistent with previous studies that reported a 
significantly shorter time taken by chest compression CPR groups when compared to conventional CPR groups. ${ }^{(14,28,29)}$ A possible explanation could be that, unlike our study, the CPR guideline used in previous studies involved the delivery of two rescue breaths before initiating compressions. An aversion to MTM rescue breathing has often been cited as a reason for participant hesitation to initiate CPR. ${ }^{(30)}$

Our study clearly demonstrated that participants who underwent simplified CPR training could perform a greater number of chest compressions with fewer interruptions compared to those who received standard CPR training. Participants performing standard CPR paused three times as long as those performing simplified CPR. During standard CPR, compressions are paused to perform MTM ventilation. However, our findings showed that $39.4 \%$ of participants in the standard CPR group did not perform MTM ventilation. A possible reason could be the 'saliva secretions' that were placed around the tested manikins' mouths. Even among participants who attempted MTM ventilation, most were unable to attain the appropriate amount of ventilation during the eight-minute test. Given the difficulties of attaining effective ventilation, which was also reported in previous studies, ${ }^{(17,24)}$ it would be reasonable to focus on chest compression for OHCA. Although giving ventilation in CPR is important for asphyxia-associated arrests, such arrests are less common than arrests with a cardiac origin in adult victims. Even with standard CPR, the chances of survival from asphyxia-associated arrest are poor. ${ }^{(8)}$ Besides MTM ventilation, the complicated method of identifying the anatomical landmarks for hand placement in standard CPR technique could have further contributed to the length of pause among participants performing standard CPR. ${ }^{(12)}$

We also showed that the simplified CPR group could deliver a higher number of adequate compressions and up to three times the proportion of adequate compressions when compared to the standard CPR group. Previous studies have shown inconsistent results, with some reporting that simplified CPR resulted in a significantly higher number or proportion of adequate compressions, ${ }^{(28,31)}$ whereas others reported that it delivered an equal, if not lower, number or proportion of adequate compressions when compared to standard CPR. ${ }^{(32)}$ According to Trowbridge et al, pausing for MTM ventilation in standard CPR could function as a 'break time' for rescuers to rest their arms, hence resulting in less rescuer fatigue and possibly promoting adequate compression. ${ }^{(32)}$ Variations in outcomes of adequate compression across studies could have been influenced by participant characteristics and the duration of tests, which have been found to be associated with rescuer fatigue. ${ }^{(31)}$ Nevertheless, given that both CPR groups in our study received an equal length of training, the reduced amount of content received by the simplified CPR group could have enabled its participants to have more time to focus on practising the proper chest compression technique. This opportunity to engage in deliberate practice is essential to achieving long-term retention of learning. ${ }^{(33)}$

Our study had several limitations. First, our participants possessed at least a minimum educational qualification of
GCE O-level, which may not be representative of the general population. Second, as testing was not done before (at baseline) and immediately after training, we did not compare participants' CPR performance at these two time-points, which may have been relevant for determining their level of retention. Third, although CPR performance was evaluated using a simulated scenario mimicking a realistic OHCA, the study outcome cannot be directly applied to a real-life setting. Lastly, there were some technical faults in the Resusci Anne ${ }^{\circledR}$ SkillReporter ${ }^{\text {TM }}$ manikins, which resulted in the failure to capture the performance of some participants, thus leading to missing data.

In conclusion, simplified CPR training for laypersons, by focusing on continuous chest compressions with simple hand placement for chest compression, could lead to better acquisition and retention of CPR algorithms, and better quality of chest compression when compared to the standard CPR programme. This easy-to-learn CPR will not only encourage the public to acquire CPR skills, but also increase their likelihood of performing it should the need arise.

\section{ACKNOWLEDGEMENTS}

The authors would like to thank the staff of Centre for Healthcare Simulation, NUS Yong Loo Lin School of Medicine, Singapore, for supporting this study. We would also like to thank Medical Publications Support Unit, National University Health System, Singapore, for providing editing services for this manuscript. This study was funded by Singapore Heart Foundation.

\section{REFERENCES}

1. Nakahara S, Tomio J, Ichikawa M, et al. Association of bystander interventions with neurologically intact survival among patients with bystander-witnessed out-of-hospital cardiac arrest in Japan. JAMA 2015; 314:247-54.

2. El Sayed MJ, Tamim H, Nasreddine Z, Dishjekenian M, Kazzi AA. Out-of-hospital cardiac arrest survival in Beirut, Lebanon. Eur J Emerg Med 2014; 21:281-3.

3. Sasson C, Meischke H, Abella BS, et al; American Heart Association Council on Quality of Care and Outcomes Research, Emergency Cardiovascular Care Committee, Council on Cardiopulmonary, Critical Care, Perioperative and Resuscitation, Council on Clinical Cardiology, and Council on Cardiovascular Surgery and Anesthesia. Increasing cardiopulmonary resuscitation provision in communities with low bystander cardiopulmonary resuscitation rates: a science advisory from the American Heart Association for healthcare providers, policymakers, public health departments, and community leaders. Circulation 2013; 127:1342-50

4. Bradley SM, Rea TD. Improving bystander cardiopulmonary resuscitation. Curr Opin Crit Care 2011; 17:219-24.

5. Vaillancourt C, Stiell IG, Wells GA. Understanding and improving low bystander CPR rates: a systematic review of the literature. CJEM 2008; 10:51-65.

6. Travers AH, Perkins GD, Berg RA, et al; Basic Life Support Chapter Collaborators. Part 3: Adult Basic Life Support and Automated External Defibrillation: 2015 International Consensus on Cardiopulmonary Resuscitation and Emergency Cardiovascular care Science with Treatment Recommendations. Circulation 2015; 132(16 Suppl 1):S51-83.

7. Perkins GD, Travers AH, Berg RA, et al. Part 3: Adult basic life support and automated external defibrillation: 2015 International Consensus on Cardiopulmonary Resuscitation and Emergency Cardiovascular Care Science with Treatment Recommendations. Resuscitation 2015; 95:e43-69.

8. Iwami T, Kitamura T, Kiyohara K, Kawamura T. Dissemination of chest compression-only cardiopulmonary resuscitation and survival after out-ofhospital cardiac arrest. Circulation 2015; 132:415-22.

9. Hüpfl M, Selig HF, Nagele P. Chest-compression-only versus standard cardiopulmonary resuscitation: a meta-analysis. Lancet 2010; 376:1552-7.

10. Finn JC, Bhanji F, Lockey A, et al; Education, Implementation, Teams Chapter Collaborators. Part 8: Education, implementation, and teams: 2015 International Consensus on Cardiopulmonary Resuscitation and Emergency Cardiovascular Care Science with Treatment Recommendations. Resuscitation 2015; 95:e203-24. 11. Lim SH, Wee FC, Chee TS.. Basic Cardiac Life Support: 2016 Singapore 
guidelines. Singapore Med J 2017; 58:347-53.

12. Handley AJ. Teaching hand placement for chest compression--a simpler technique. Resuscitation 2002; 53:29-36.

13. Lim SH, Aw SJ, Cheong MA, et al. A randomised control trial to compare retention rates of two cardiopulmonary resuscitation instruction methods in the novice. Resuscitation 2016; 103:82-7.

14. Heidenreich JW, Sanders AB, Higdon TA, et al. Uninterrupted chest compression CPR is easier to perform and remember than standard CPR. Resuscitation 2004; 63:123-30.

15. Chamberlain DA, Hazinski MF; European Resuscitation Council; American Heart Association; Heart and Stroke Foundation of Canada; Resuscitation Council of Southern Africa; Australia and New Zealand Resuscitation Council; Consejo Latino-Americano de Resusucitación. Education in resuscitation: An ILCOR Symposium: Utstein Abbey: Stavanger, Norway: June 22-24, 2001. Circulation 2003; 108:2575-94.

16. Van Merriënboer JJ, Sweller J. Cognitive load theory in health professional education: design principles and strategies. Med Educ 2010; 44:85-93.

17. Nishiyama C, Iwami T, Kawamura T, et al. Effectiveness of simplified ches compression-only CPR training for the general public: a randomized controlled trial. Resuscitation 2008; 79:90-6

18. Ashton A, McCluskey A, Gwinnutt CL, Keenan AM. Effect of rescuer fatigue on performance of continuous external chest compressions over $3 \mathrm{~min}$. Resuscitation 2002; 55:151-5.

19. Heidenreich JW, Bonner A, Sanders AB. Rescuer fatigue in the elderly: standard vs. hands-only CPR. J Emerg Med 2012; 42:88-92.

20. Polit DF, Beck CT. Essentials of nursing research: appraising evidence for nursing practice. 8th ed. Philadelphia: Wolters Kluwer Health, 2010.

21. National Resuscitation Council, Singapore. Guidelines for accreditation of citizen cardiopulmonary resuscitation + automated external defibrillation training centre (citizen cpr+AED TC) [online]. Available at: https://www.nhcs. com.sg/educationandtraining/cprtrainingaccreditationguidelines/cprtraining/ Documents/Microsoft\%20Word\%20-\%20Citizen\%20CPR+AED_TC_ Guidelines-21\%20Feb\%202010.pdf. Accessed March 29, 2018.

22. Whitfield RH, Newcombe RG, Woollard M. Reliability of the Cardiff Test of basic life support and automated external defibrillation version 3.1. Resuscitation 2003; 59:291-314
23. Hong DY, Park SO, Lee KR, Baek KJ, Shin DH. A different rescuer changing strategy between 30:2 cardiopulmonary resuscitation and hands-only cardiopulmonary resuscitation that considers rescuer factors: a randomised cross-over simulation study with a time-dependent analysis. Resuscitation 2012; 83:353-9.

24. Williams JG, Brice JH, De Maio VJ, Jalbuena T. A simulation trial of traditional dispatcher-assisted CPR versus compressions-only dispatcher-assisted CPR. Prehosp Emerg Care 2006; 10:247-53.

25. Swor R, Compton S, Vining F, et al. A randomized controlled trial of chest compression only CPR for older adults-a pilot study. Resuscitation 2003; 58:177-85.

26. Chamberlain DA, Smith A, Colquhoun M, et al. Randomised controlled trials of staged teaching for basic life support: 2. Comparison of CPR performance and skill retention using either staged instruction or conventional training. Resuscitation 2001; 50:27-37.

27. Riegel B, Wik L, Doherty A. Training adults to perform cardiopulmonary resuscitation. In: Ornato JP, Peberdy MA, eds. Cardiopulmonary Resuscitation. New York: Humana Press, 2005: 199-210.

28. Dias JA, Brown TB, Saini D, et al. Simplified dispatch-assisted CPR instructions outperform standard protocol. Resuscitation 2007; 72:108-14.

29. Dorph E, Wik L, Steen PA. Dispatcher-assisted cardiopulmonary resuscitation. An evaluation of efficacy amongst elderly. Resuscitation 2003; 56:265-73.

30. Nishiyama C, Iwami T, Kitamura T, et al. Long-term retention of cardiopulmonary resuscitation skills after shortened chest compression-only training and conventional training: a randomized controlled trial. Acad Emerg Med 2014; 21:47-54.

31. Heidenreich JW, Berg RA, Higdon TA, et al. Rescuer fatigue: standard versus continuous chest-compression cardiopulmonary resuscitation. Acad Emerg Med 2006; 13:1020-6

32. Trowbridge C, Parekh JN, Ricard MD, et al. A randomized cross-over study of the quality of cardiopulmonary resuscitation among females performing 30:2 and hands-only cardiopulmonary resuscitation. BMC Nurs 2009; 8:6

33. Ericsson KA. Deliberate practice and the acquisition and maintenance of expert performance in medicine and related domains. Acad Med 2004; 79(10 Suppl):S70-81. 